\title{
NICE - New Ideal Coset Encryption -
}

\author{
Michael Hartmann ${ }^{1}$, Sachar Paulus ${ }^{2}$, and Tsuyoshi Takagi ${ }^{3}$ \\ 1 Darmstadt University of Technology, \\ Alexanderstr. 10, 64283 Darmstadt, Germany, \\ mhartman@cdc.informatik.tu-darmstadt.de, \\ 2 SECUDE Sicherheitstechnologie Informationssysteme GmbH, \\ Landwehrstr. 50a, 64283 Darmstadt, Germany, \\ paulus@secude.com, \\ 3 NTT Information Sharing Platform Laboratories, \\ Immermannstr. 40, D-40210 Düsseldorf, Germany, \\ ttakagi@ntt.de
}

\begin{abstract}
Recently, a novel public-key cryptosystem constructed on number fields is presented. The prominent theoretical property of the public-key cryptosystem is a quadratic decryption bit complexity of the public key, which consists of only simple fast arithmetical operations. We call the cryptosystem NICE (New Ideal Coset Encryption). In this paper, we consider practical aspects of the NICE cryptosystem. Our implementation in software shows that the decryption time of NICE is comparably as fast as the encryption time of the RSA cryptosystem with $e=2^{16}+1$. To show if existing smart cards can be used, we implemented the NICE cryptosystem using a smart card designed for the RSA cryptosystem. Our result shows that the decryption time of NICE is comparably as fast as the decryption time of RSA cryptosystem but not so fast as in software implementation. We discuss the reasons for this and indicate requirements for smartcard designers to achieve fast implementation on smartcards.
\end{abstract}

Key words: public-key cryptosystem, fast decryption, quadratic order, smart card implementation.

\section{Why NICE?}

Plenty of public-key cryptosystem not relying only on the RSA cryptosystem have been proposed. They are stemming from deep number theory (hyper- and superelliptic curves) to geometry and combinatorics (LLL-based systems). One major advantage of RSA is its simplicity: it can be easily implemented, one only needs a moderate background in mathematics to understand it. Moreover, RSA is quite fast - there exist public key cryptosystems which are much faster, but the combination of simplicity, speed and confidence in its security makes it the most practical cryptosystem. Until now, only one other system may be considered as equally interesting: ElGamal type systems on elliptic curves. Elliptic curves are somewhat more complicated, and the best known algorithms to break elliptic curve cryptosystems are much slower, in the order of exponential complexity. 
So, both speed and security are worth being paid by the higher mathematical complexity.

But: both systems have one drawback in common: the decryption/signing time is of cubic complexity in the bit length of the public key for both systems because these steps consist of modular multiplication(s). This becomes even more important when thinking of smart cards. The smart card is considered to be the personal security computing device of tomorrow. It contains all personal secret information, especially the private keys for public key systems like decryption and signing. The complexity of PC operating systems is too high to be reliably secure; every relevant security operation should be effected by the smart card. Non-relevant operations like public key encryption and signature verification could be done by the PC. Operations which cannot be transferred to the PC at all due to security reasons are decryption and signing. Considering RSA, the task which has to be effected by a low power computing device is precisely the most complex task. Moreover, we can expect that the key length of the public key will increase with the progress of hardware technology. In addition, there are no guarantees that new sub-exponential attacks for the basic number theoretic problem will not be suddenly proposed. Therefore it would be better to have a more efficient public key cryptosystem.

As an alternative, we might use a new public-key cryptosystem constructed over number fields [18]. The cryptosystem has a theoretically fast decryption process such as a quadratic decryption complexity of bit-length of a public-key, which consists of only simple fast arithmetical operations. So even if the key length gets bigger in the future, there will be no great increase of the computational complexity. This becomes even more important when thinking of smart cards. In this paper, we call the new cryptosystem NICE, (New Ideal Coset Encryption). We focus on the practical aspects of NICE cryptosystem. We implement the NICE cryptosystem over different architectures, namely software on a standard PC and on a smartcard designed for the RSA cryptosystem. Our implementation in software shows NICE is as fast as the encryption time of the RSA cryptosystem with $e=2^{16}+1$. Implementation on a smartcard designed for the RSA cryptosystem is comparably as fast as the decryption time of the RSA cryptosystem but not so fast as in software implementation. We discuss the reasons for this and indicate requirements to achieve fast implementation on smartcards.

This paper is organized as follows: In section 2, we give several applications based on NICE cryptosystem. In section 3, we explain the details of the algorithms of the NICE cryptosystem. In section 4, we show timings of the implementation in software. In section 5 , we discuss a smart card implementation and its problems.

\section{Applications}

In section 3, we will present NICE in the formulation of an encryption scheme. An immediate application is therefore session key distribution from a powerful server 
to a device which has a limited computing power or where time is important. An example for such a device is e.g. a mobile phone.

Another application of NICE is the use as an authentication scheme. The usual protocols (2-way and 3-way) can be adapted to use NICE as encryption component. Of course, it could be combined with RSA, where the modulus is the absolute value of the discriminant of the non-maximal order, i.e. $\Delta_{q}$. In that case, the 3 -way protocol can be realized in such a way that the client (= the low computing power device) only effects the fast components of both algorithms.

As a last application, we propose an undeniable signature scheme. NICE itself cannot be used as a classical signature scheme; undeniable signature schemes have their own use e.g. in online transactions. A signature of this kind cannot be verified without the interaction of the signer. The standard example for its application is its use by a software development company: the distributed software is signed by means of an undeniable signature of the company to allow legal users to ensure themselves that they use unmodified software. Since interaction with the seller is needed to check the signature, illegal users either cannot check and risk to use some virus-infected software or will be traced by the software-seller as soon as they ask for interactive verification. Details can be found in [4]. Again, the low computing power device only effectuates the NICE decryption steps, so smart cards can be used for assuring the security of online transactions.

\section{The NICE Cryptosystem}

In this section, we present an overview of the NICE cryptosystem. Details can be found in [18]. The idea of NICE is roughly as follows: consider two finite abelian groups $G$ and $H$ which are related by a surjective map $\pi: G \rightarrow H$. Moreover, there exists a well-defined bijective mapping of sets $\phi: H \rightarrow U$ of $H$ onto a subset of $U$ of $G$ such that $\pi(\phi(M)))=M$ for all $M \in H$. The representation of elements of $G$ and the group operation algorithm of $G$ are publicly known, as well as an element $h$ of the kernel of $\pi$. $U$ is chosen such that a consecutive set of representations of elements of $G$ are representations of elements of $U$. This information is publicly known. Assume that you know the group $H$ (i.e. representation of group elements and group operation) and how to compute $\pi$, but no one else does. The message space consists of the publicly known elements of $U$. Now, a message $m$ is probabilistically encrypted by randomly multiplying an element $h^{r}$ of $\operatorname{Ker} \pi$ onto it: the ciphertext is $c=m * h^{r}$. Decryption simply works as follows: compute $\phi\left(\pi\left(m * h^{r}\right)\right)$.

This is a secure cryptosystem if the computation of the map $\pi$ cannot be deduced from the given information, namely the group $G$, the kernel element $h$ and the test for $U$. There exist some constructions of this scheme using number theoretic problems, e.g. [17]. An overview can be found in [19].

The following implementation of this scheme is especially interesting: Generate two random primes $p, q>4$ such that $p \equiv 3(\bmod 4)$ and let $\Delta_{1}=-p$. Let $H=C l\left(\Delta_{1}\right)$ be the ideal class group of the maximal order with discriminant $\Delta_{1}$ and $G=C l\left(\Delta_{q}\right)$ be the ideal class group of the non-maximal order with 
conductor $q . \Delta_{q}$ will be public, whilst its factorization into $\Delta_{1}$ and $q$ will be kept private.

Any element of $G$ is given by a pair of numbers $(a, b)$ such that $0<a \leq$ $\sqrt{\left|\Delta_{q}\right| / 3},-a<b \leq a$ and $b^{2} \equiv \Delta_{q} \bmod 4 a$ (and some other minor requirements). Elements of $H$ are represented in the same way with $\Delta_{1}$ instead of $\Delta_{q}$. The group operation works as follows:

Composition in $\mathrm{Cl}\left(\Delta_{q}\right)$

Input: $\left(a_{1}, b_{1}\right),\left(a_{2}, b_{2}\right) \in \mathrm{Cl}\left(\Delta_{q}\right)$, the discriminant $\Delta_{q}$

Output: $(a, b)=\left(a_{1}, b_{1}\right) *\left(a_{2}, b_{2}\right)$.

\section{1. / Multiplication step */}

1.1. Solve $d=u a_{1}+v a_{2}+w\left(b_{1}+b_{2}\right) / 2$ for $d, u, v, w \in \mathbb{Z}$ using the extended Euclidean algorithm

1.2. $a \leftarrow a_{1} a_{2} / d^{2}$

1.3. $b \leftarrow b_{2}+\left(v a_{2}\left(b_{1}-b_{2}\right)+w\left(\Delta_{q}-b_{2}^{2}\right) / 2\right) / d \bmod 2 a$

$2 / *$ Reduction step */

2.1. $c \leftarrow\left(\Delta_{q}-b^{2}\right) / 4 a$

2.2. WHILE $\{-a<b \leq a<c\}$ or $\{0 \leq b \leq a=c\}$ DO

2.2.1. Find $\lambda, \mu \in \mathbb{Z}$ s.t. $-a \leq \mu=b+2 \lambda a<a$ using division with remainder

2.2.2. $(a, b, c) \leftarrow\left(c-\lambda \frac{b+\mu}{2},-\mu, a\right)$

2.3. IF $a=c$ AND $b<0$ THEN $b \leftarrow-b$

2.4. RETURN $(a, b)$

This algorithm has quadratic bit complexity $O\left(\left(\log _{2} \Delta_{q}\right)^{2}\right)$ and is only needed for the encryption step. For the decryption step, we need only to compute $\pi$. The computation of the map $\pi$ works as follows:

\section{Computation of $\pi$}

Input: $(a, b) \in C l_{\Delta_{q}}$, the fundamental discriminant $\Delta_{1}$, the discriminant $\Delta_{q}$ and the conductor $q$

Output: $(A, B)=\pi((a, b))$.

1. $b_{\mathcal{O}} \leftarrow \Delta_{q} \bmod 2$

2. Solve $1=u q+v a$ for $u, v \in \mathbb{Z}$ using the extended Euclidean algorithm

3. $B \leftarrow b u+a b_{\mathcal{O}} v \bmod 2 a$

4. $C \leftarrow\left(\Delta_{1}-B^{2}\right) / 4 A$

5. WHILE NOT $(\{-A<B \leq A<C\}$ or $\{0 \leq B \leq A=C\})$ DO

5.1 Find $\lambda, \mu \in \mathbb{Z}$ s.t. $-A \leq \mu=B+2 \lambda A<A$ using division with remainder

$5.2(A, B, C) \leftarrow\left(C-\lambda \frac{B+\mu}{2},-\mu, A\right)$

6. IF $A=C$ AND $B<0$ THEN $B \leftarrow-B$

7. $\operatorname{RETURN}(A, B)$

This algorithm $\pi$ has quadratic bit complexity $O\left(\left(\log _{2} \Delta_{q}\right)^{2}\right)([18])$. Moreover, only simple well-known operations are needed, thus this algorithm can easily be implemented on an existing smart card. 
Finally, the test belonging to $U$ is simply whether for an element $(a, b) a$ is smaller than $\left[\sqrt{\left|\Delta_{1}\right| / 4}\right]$ (or a lower bound thereof of the form $2^{k}$ ). The computation of the map $\phi$ will not be needed in that implementation.

We explain how the NICE encrytion scheme works: In the key generation, we choose an element $\left(h, b_{h}\right)$ from the kernel $\operatorname{Ker} \pi$ and make $\left(h, b_{h}\right)$ public. The message $m$ is embedded in an element $\left(m, b_{m}\right)$ of $C l\left(\Delta_{q}\right)$ with $m$ smaller than $\left[\sqrt{\left|\Delta_{1}\right| / 4}\right]$. Encryption is done in the class group $C l\left(\Delta_{q}\right)$ by computing $\left(c, b_{c}\right)=\left(\left(m, b_{m}\right) *\left(h, b_{h}\right)^{r}\right)$, where $r$ is a random integer smaller than $2^{l}$ with $l=$ $\log _{2}\left(q-\left(\frac{\Delta_{1}}{q}\right)\right)$. Then, having the secret information, namely the knowledge of the conductor $q$, one can go to the maximal order and the image of the message $\left(m, b_{m}\right)$ in the maximal order is revealed, since $\pi\left(c, b_{c}\right)=\pi\left(m, b_{m}\right)$ and $m$ can be recovered without computing $\phi$.

\section{The NICE encryption protocol}

1. Key generation: Generate two random primes $p, q>4$ with $p \equiv 3(\bmod 4)$ and $\sqrt{p / 4}<q$. Let $\Delta_{1}=-p$ and $\Delta_{q}=\Delta_{1} q^{2}$. Let $k$ and $l$ be the bit lengths of $\left[\sqrt{\left|\Delta_{1}\right| / 4}\right]$ and $q-\left(\frac{\Delta_{1}}{q}\right)$ respectively. Choose an element $\left(h, b_{h}\right)$ in $C l\left(\Delta_{q}\right)$, where

$$
\pi\left(\left(h, b_{h}\right)\right)=(1,0)
$$

Then $\left(\left(h, b_{h}\right), \Delta_{q}, k, l\right)$ are the system parameters, and $q$ is the secret key.

2. Encryption: Let $\left(m, b_{m}\right)$ be the plaintext, in $C l\left(\Delta_{q}\right)$ with $\log _{2} m<k$. Pick up a random $l-1$ bit integer and we encrypt the plaintext as follows using binary exponentiation and precomputation techniques:

$$
\left(c, b_{c}\right)=\left(m, b_{m}\right) *\left(h, b_{h}\right)^{r}
$$

Then $\left(c, b_{c}\right)$ is the ciphertext.

3. Decryption: Using the secret key $q$, we compute $\left(d, b_{d}\right)=\pi\left(\left(c, b_{c}\right)\right)$. The plaintext is then $m=d$.

A message embedding technique and security aspects of this cryptosystem, we refer to [18]. Again, please note that this cryptosystem can easily be implemented using well-known techniques and existing smart cards. This will be shown in the next section.

\section{NICE Running Times in Software}

The prominent property of the proposed cryptosystem is the running time of the decryption. Most prominent cryptosystems require decryption time $O\left(\left(\log _{2} n\right)^{3}\right)$, where $n$ is the size of the public key. The total running time of the decryption process of our cryptosystem is $O\left(\left(\log _{2} \Delta_{q}\right)^{2}\right)$ bit operations. In order to demonstrate the improved efficiency of our decryption, we implemented our scheme using the LiDIA library [2]. It should be emphasized here that our implementation was not optimized for cryptographic purposes - it is only intended to 
provide a comparison between RSA and NICE. The results are shown in table 1 . In these tests, we did choose $p \approx q \approx n^{1 / 3}$, so that breaking RSA and NICE by factoring is approximately equally hard. Other variations and a discussion of the variants can be found in [18].

Table 1. Average timings for the new cryptosystem with 80-bit encryption exponent $r$ compared to RSA with encryption exponent $2^{16}+1$ over 100 randomly chosen pairs of primes of the specified size on a Pentium $266 \mathrm{Mhz}$ using the LiDIA library

\begin{tabular}{|l|c|c|c|c|}
\hline $\log _{2}\left(\Delta_{q}\right)$ & 1024 & 1536 & 2048 & 3072 \\
\hline RSA encryption & $2.2 \mathrm{~ms}$ & $4.8 \mathrm{~ms}$ & $7.5 \mathrm{~ms}$ & $15.2 \mathrm{~ms}$ \\
RSA classical decryption & $259.2 \mathrm{~ms}$ & $751.3 \mathrm{~ms}$ & $1643.9 \mathrm{~ms}$ & $4975.6 \mathrm{~ms}$ \\
RSA decryption with CRT & $110.5 \mathrm{~ms}$ & $291.7 \mathrm{~ms}$ & $629.7 \mathrm{~ms}$ & $1855.5 \mathrm{~ms}$ \\
\hline NICE encryption & $602.9 \mathrm{~ms}$ & $1180.1 \mathrm{~ms}$ & $1902.0 \mathrm{~ms}$ & $3933.5 \mathrm{~ms}$ \\
NICE decryption & $3.8 \mathrm{~ms}$ & $6.2 \mathrm{~ms}$ & $10.0 \mathrm{~ms}$ & $19.3 \mathrm{~ms}$ \\
\hline
\end{tabular}

Observe that one can separate the fast exponentiation step of the encryption as a "precomputation" stage. Indeed, if we can securely store the values $\left(p, b_{p}\right)^{r}$, then the actual encryption can be effected very rapidly, since it requires only one ideal multiplication and one ideal reduction. Moreover, using well-known techniques for randomized encryption, we can even reduce the encryption time much more. Note that no square root technique like the Pollard-rho method or Shanks' algorithm are directly applicable to the ciphertext $\left(c, b_{c}\right)$, because the encryption consists of $\left(c, b_{c}\right)=\left(m, b_{m}\right)\left(p, b_{p}\right)^{r}$ where $r$ is a random exponent and $\left(m, b_{m}\right)$ is the secret plaintext. This means that we can use a very short random exponent $r$ having e.g. about 80 bits.

It should be mentioned that the size of a message for our cryptosystem is significantly smaller than the size of a message for the RSA encryption (e.g. 256 bit vs. 768 bit, or 341 bit vs. 1024 bit). In connection with the very fast decryption time, an excellent purpose for our cryptosystem could be (symmetric) key distribution. In that setting, the short message length is not a real drawback. On the other hand, the message length is longer than for ElGamal encryption on "comparably" secure elliptic curves (e.g. 341 bit vs. 180 bit).

Table 2. Rate of the speed increasing when the bit-length of a public-key becomes larger

\begin{tabular}{|l|c|c|c|c|}
\hline $\log _{2}(n)$ & 1024 & 1536 & 2048 & 3072 \\
\hline RSA encryption & 1 & 2.18 & 3.41 & 6.91 \\
RSA classical decryption & 1 & 2.90 & 6.34 & 19.20 \\
RSA decryption with CRT & 1 & 2.64 & 5.70 & 16.79 \\
\hline NICE encryption & 1 & 1.96 & 3.15 & 6.52 \\
NICE decryption & 1 & 1.63 & 2.63 & 5.08 \\
\hline
\end{tabular}


Note that even if a public-key becomes large, the rate at which the speed of decryption of NICE increases is not so large as that of the RSA cryptosystem. This shows the effectiveness of a quadratic decryption time of NICE cryptosystem. The ratio is given in table 2 .

\section{A Smart Card Implementation and Its Problems}

Moreover, we implemented the NICE decryption on a smart card. More precisely, we implemented the NICE decryption algorithm using the Siemens development kit for chip card controller ICs based on Keil PK51 for Windows. As assembler we used A51, as linker L51 to generate code for the 8051 microcontroller family. The software simulation were made using dScope-51 for Windows and the drivers for SLE 66CX160S. Thus, we realized a software emulation of an assembler implementation of the NICE decryption algorithm to be run on the existing Siemens SLE 66CX160S. Unfortunately, the timings of this software simulation were unrealistic. So, we did run some timings on a hardware simulator for SLE 66CX160S. Thanks to Deutsche Telekom AG, Produktzentrum Telesec in Siegen and Infineon/Siemens in Munich for letting us use their hardware simulator. See the timings for decryption in table 3 for a smart card running at $4.915 \mathrm{MHz}$.

Table 3. Timings for the decryption of the new cryptosystem compared to RSA using the hardware simulator of Siemens 66CX160S at $4.915 \mathrm{MHz}$

\begin{tabular}{|l|c|}
\hline $\log _{2}(n)$ & 1024 \\
\hline RSA decryption with CRT & $490 \mathrm{~ms}$ \\
\hline New CS decryption for $p \approx q \approx \Delta_{q}^{1 / 3}$ & $1242 \mathrm{~ms}$ \\
\hline Improved version & $1035 \mathrm{~ms}$ \\
\hline
\end{tabular}

The very first implementation was very inefficient; the straightforward algorithms used in the software comparison proved to be much slower on the smart card than the existing RSA on the card. This was surprising, but after a while this could be easily explained: the cryptographic coprocessor has been optimized for modular exponentiation. On the other side, NICE uses mostly divisions with remainder and comparisons. These operations are slow on the coprocessor, so we had to modify the decryption algorithm to speed it up in hardware. We describe here two significant changes:

The computation of the inverse of $a$ modulo $q$ (step 3 in the computation of $\pi$ ) using the extended Euclidean algorithm took (with $q \approx p \approx 341$ bit) about 9 seconds (!), whereas computing the inverse using Fermat's little theorem by using fast exponentiation mod $q$ - took less than 1 second. Note that the decryption time using this method is no longer of quadratic complexity.

In the reduction process the quotient in the division with remainder step is most of the time very small (say $\leq 10$, see Appendix A); to effect a division is 
this case is much more time consuming than subsequent subtractions. We did replace reduction step 5

5.1 Find $\lambda, \mu \in \mathbb{Z}$ s.t. $-A \leq \mu=B+2 \lambda A<A$ using division with remainder $5.2(A, B, C) \leftarrow\left(C-\lambda \frac{B+\mu}{2},-\mu, A\right)$

by the following algorithm.

\subsection{WHILE $B \leq-A$ OR $B>A$ DO}

5.1.1 IF $B<0$ THEN $B \leftarrow B+2 A ; C \leftarrow C-(B+A)$;

5.1.2 ELSE $B \leftarrow B-2 A ; C \leftarrow C-(B-A)$;

Every time that the bitlength of $B$ was exceeding the bitlength of variant $B$ by at least 3 . Using this improvement, we could decrease the running time from about $1.8 \mathrm{~s}$ to $1.2 \mathrm{~s}$. This is already faster than a 1024 bit RSA decryption without Chinese remainder theorem (approx. $1.6 \mathrm{~ms}$ ).

The timings in table 3 were made including these two improvements. Moreover, a detailed timing analysis in Siegen showed that both our static memory management and as well as the cryptographic coprocessor are not optimal for this algorithm. We discuss this in the sequel. An average overview of the most time consuming parts is given in table 4 .

Table 4. Detailed timings for different functions in the decryption of the new cryptosystem

\begin{tabular}{|l|c|}
\hline Function & Average time over the whole computation \\
\hline mul, multiplications on the coprocessor & $170 \mathrm{~ms}$ \\
div, divisions on the coprocessor & $231 \mathrm{~ms}$ \\
left_adjust, length correction of the variables & $376 \mathrm{~ms}$ \\
C2XL, moving numbers into the coprocessor & $118 \mathrm{~ms}$ \\
XL2C, moving numbers out of the coprocessor & $223 \mathrm{~ms}$ \\
others (comparisons, small operations) & $114 \mathrm{~ms}$ \\
\hline Overall time & $1242 \mathrm{~ms}$ \\
\hline
\end{tabular}

One major difference between RSA and NICE is the number of variables needed during the computation of the decryption algorithm. In our implementation, we need to store 11 variables of length at most 2048 bit. Computations of the cryptographic coprocessor are shortening these variables. Thus, we had to adjust the length of the variables after each important operation. This was done by moving the top nonzero bytes of the number to the fixed address of the number and so "erasing" leading zero bytes. To do this, we used the cryptographic coprocessor. Now the exact timings showed that about $33 \%$ of the running time is spent by the function left_adjust, which effects this correction.

Now changing the memory management from static to dynamic (i.e. in the XL2C and C2XL functions making the appropriate changes and having additionally some registers holding the starting address of the numbers), we got an 
improvement to $\mathbf{6 3 7} \mathbf{~ m s}$ using the software simulator. Both Infineon/Siemens and Deutsche Telekom reported the overall time of the hardware simulator now to be $\mathbf{1 0 3 5} \mathbf{m s}$. Note that the amount of memory required is $\mathbf{9 6 5}$ Bytes and thus fits into a real SLE 66CX160S.

As one can see from table 4, another important time consuming operation is to move numbers into and out of the coprocessor. At this point, we would get a speedup of about $350 \mathrm{~ms}$ if we could leave the numbers in registers inside the coprocessor. It is clear that the currently used processor is not prepared for such operations, since it is optimized for RSA, thus operations with very few variables. At this point we ask the hardware community to present solutions to this problem.

\section{Conclusion and Acknowledgements}

The NICE cryptosystem is fast and well suited for software implementation. To get an equally fast speedup compared to RSA on a smart card, we think that the underlying hardware must be developed adequately. Nevertheless, if this is done, the think that NICE can be a competitor to RSA whenever fast decryption is needed.

We thank Deutsche Telekom AG, Produktzentrum Telesec for letting us testing NICE on the hardware simulator and Siemens AG/Infineon GmbH for their valuable help concerning the use of the development kit as well as running our code on their hardware simulator.

\section{References}

1. L. M. Adleman and K. S. McCurley, "Open problems in number theoretic complexity, II" proceedings of ANTS-I, LNCS 877, (1994), pp.291-322.

2. I. Biehl, J. Buchmann, and T. Papanikolaou. LiDIA - A library for computational number theory. The LiDIA Group, Universität des Saarlandes, Saarbrücken, Germany, 1995.

3. I. Biehl and J. Buchmann; "An analysis of the reduction algorithms for binary quadratic forms," Technical Report No. TI-26/97, Technische Universität Darmstadt, (1997).

4. I. Biehl and S. Paulus and T. Takagi, "Efficient Undeniable Signature Schemes based on Ideal Arithmetic in Quadratic Orders," in preparation.

5. J. Buchmann and H. C. Williams; "A key-exchange system based on imaginary quadratic fields," Journal of Cryptology, 1, (1988), pp.107-118.

6. J. Buchmann and H. C. Williams; "Quadratic fields and cryptography," London Math. Soc. Lecture Note Series 154, (1990), pp.9-26.

7. J. Buchmann, S. Düllmann, and H. C. Williams. On the complexity and efficiency of a new key exchange system. In Advances in Cryptology - EUROCRYPT '89, volume 434 of Lecture Notes in Computer Science, pages 597-616, 1990.

8. J. Cowie, B. Dodson, R. Elkenbracht-Huizing, A. K. Lenstra, P. L. Montgomery, J. Zayer; "A world wide number field sieve factoring record: on to 512 bits," Advances in Cryptology - ASIACRYPT '96, LNCS 1163, (1996), pp.382-394. 
9. D. A. Cox: Primes of the form $x^{2}+n y^{2}$, John Wiley \& Sons, New York, 1989

10. W. Diffie and M. Hellman, "New direction in cryptography," IEEE Transactions on Information Theory, 22, (1976), pp.472-492.

11. ECMNET Project; http://www.loria.fr/ zimmerma/records/ecmnet.html

12. T. ElGamal, "A public key cryptosystem and a signature scheme based on discrete logarithm in $G F(p)$," IEEE Transactions on Information Theory, 31, (1985), pp.469-472.

13. D. Hühnlein, M. J. Jacobson, Jr., S. Paulus, and T. Takagi; "A cryptosystem based on non-maximal imaginary quadratic orders with fast decryption," Advances in Cryptology - EUROCRYPT '98, LNCS 1403, (1998), pp.294-307.

14. H. W. Lenstra, Jr., "Factoring integers with elliptic curves", Annals of Mathematics, 126, (1987), pp.649-673.

15. A. K. Lenstra and H. W. Lenstra, Jr. (Eds.), The development of the number field sieve. Lecture Notes in Mathematics, 1554, Springer, (1991).

16. A. J. Menezes, P. van Oorschot, S. Vastone: Handbook of applied crytpography. CRC Press, 1996.

17. T. Okamoto and S. Uchiyama, "A new public-key cryptosystem as secure as factoring," Advances in Cryptology - EUROCRYPT '98, LNCS 1403, (1998), pp.308-318.

18. S. Paulus and T. Takagi, "A new public-key cryptosystem over the quadratic order with quadratic decryption time," to appear in Journal of Cryptology.

19. S. Paulus and T. Takagi, "A generalization of the Diffie-Hellman problem based on the coset problem allowing fast decryption ," Proceedings of ICISC'98, Seoul, Korea, 1998.

20. R. Peralta and E. Okamoto, "Faster factoring of integers of a special form," IEICE Trans. Fundamentals, Vol.E79-A, No.4, (1996), pp.489-493.

21. R. J. Schoof: Quadratic Fields and Factorization. In: H.W. Lenstra, R. Tijdeman, (eds.): Computational Methods in Number Theory. Math. Centrum Tracts 155. Part II. Amsterdam, 1983. pp. 235-286.

\section{A An Example of the Reduction Step}

Input: discriminant $\Delta_{1}$, an ideal $\mathfrak{A}=(A, B)$ before the reduction step

$D 1=-3919553298811157368475523990994777486712879620160636686074980926686635774565281654416535613225312452663$

$A=1470919698723621747031034479033655458221345099558043935477761485226131553543707299391756504474531255189264719094757$ 659808191301659419506155735441417414491

$\mathrm{B}=-1612078661117333293617543382531888102723531401772217996101303796600583164587338163291108859457317212840954820324227$ 661206969587778502329803762655721150213

Reduction step 5.1.: quotient $\lambda$, remainder $\mu$ such that $B=2 A \lambda+\mu$

\footnotetext{
(quotient, remainder) $=$

$(-1, \quad 1329760736329910200444525575535422813719158797343869874854219173851679942500076435492404149491745297537574661786528$ $7658409413015540336682507708227113678769)$

$(-2, \quad-127612435903271747284203940962563047288319779794024205168731345688046856992818597251036644668758126413199147109490$ $159283556463934886789503896413069632105)$

$(5, \quad-785282900112624573884568439413292485034544440747434937937831117600068351530406923212045847152018597171039409030826$ $1868661757907073551937411599271404065)$

(3, 102448189335108917873708106129511177370194888095368169144208661124365741713523215381199419560853590747848324434047 $4403297520916097275928966085178754669)$

$(-2, \quad-1022412984559014750138236511849426625202189309224462971206105890906868085219738761438688942269542228647930772313523$ $574165905118338106550708986943292605)$

$(5, \quad-111052964327862543248014454108681029972621611840532971461250154942477651873609548116068054690931838077804710928090$ $93473562855601861999949481990650315)$

( $2, \quad 224721768750757491766093041444587045099908536747902569504729305667589920231890466102920134589953378731011273003071$ 265369685205138129287433281611151)

$(-24,-193997682622386160747509256203459457001302400743643940157769391868127988127520988615996593055854386819648159017406$ $4685369029295289498715467235503)$

$(5, \quad-872140247558161527297502021585254450313036153689333922978271792128743615045351839413078660868168505225167354418491$
} 
91048830504132818556443763927)

(5, -658925615422042834876348480484029082058507054052681386209011509962105060677563908394232616059830404811010727301359 $3512069684208466784180774933)$

$(3, \quad-3537254096503620593414134057544050452333307165981859271665819380718315946782301163388529333339930067189465564000769$ 287233896579646995020989815)

(7, -247222284294593727142489621540238650242932541449160633234337068881461071113337993384784406154327323546383475378203 $76474826892936909497877111)$

$(2, \quad 211238960823671342461903216289647740521689040736096428971091833115479874530277048960194279957267650917082000952992$ $7566081981468920686945135)$

$(-5,-138828499610837630682998254650680087729785399344104356860281267036927461979957572311938410081908330045415812611779$ 611796816538719307405505)

(3, - -7658114509885547423144046883303894668462310641251449515590759557414197782566477229459016888940284991233814769828608 $3138580548663993732859)$

$(6, \quad 451715975973337636239923655622704513018369560900086413869204962835152615559671858853242965579677357251079839392053$ $844630453417984392931)$

$(-3, \quad 579493992911536599517350321866083438435686742562973463372479826459392480226779856939226160460117478967847924671753$ 92968596489710481213)

$(-3, \quad 135067752525885105483994559664721938559177175680541689433450130455304919692737374065720006258404545809371465593318$ 9049738778828983289)

$(-15, \quad 337193319312295431745969016664643151387915479765592068235657385273215560443110982269888116148705401309431343138225$ 96913769977980851)

$(-3, \quad 323878473831343845968960745512074363754506782760808676681774080723182382322942699332100336805150642284551592326904$ 4296100266373941)

$(-4, \quad 167144197381464431967528225738718542314051660172099527690831373123590316239808571782330789831021939891807455958096$ 732542876200243)

$(-5, \quad-309390011597559593159761073745581970668200194635499292322116436596067679796830203942404937063400931822640702431265$ 3906939082883)

$(11, \quad-115310685805821673604505436475263765545172934158683202462769686123589664701441798228728715241781657550421817808920$ 121862424193)

$(3, \quad-214164778849294341813886319240237413612645988098168730763311338303186025963320880697339330474638842906957278383121$ 52170109453)

(2, $\quad 128885235911603341061379124399416424159190535507373563424716539280561837794601490501570150868125552599247818531014$ 6905542061)

$(-8, \quad 316335781594392130649537991526978632041159226767264341022335809622254943347009615572076976512856499763471626857986$ 37456771)

$(-5, \quad-132100188970518808134989273741019953395958169925083468958496091548029282207578810138129189350877886346393515471619$ 9449891)

(5, - -11820767885517306027346197667704751166610749535623823332585074677744843339528870419622234444687351217744461329878842 257829)

$(2, \quad 211192794617132775962070178935022665383131433119630308575044151078117101318282538223456317584240935159085658194389$ 75697)

$(-2, \quad-274328530511417242554111054080256110572002014193891842660039774575578405921937962321580147605536058202955477454722$ 6713)

$(3, \quad 286074815889198540147442971313659278227472798937810428709777362705663473916998086767681817465542781829132249359517$ 975)

$(-3, \quad 136753299791205246544364486899154151347754882077985198546727042251978367689138220757139643994263442612011028174033$ 09)

$(-7, \quad-5733925658197840890157616477656092654752132021299440670896385313615178397931288947596754116299993866769288097157573)$

(3, 464201412472028996327587354924283084916556650187378596448769488246653936727190431310329578844089903814145126014811)

$(-4, \quad 2648752977947845396817832353867014934646503864836612394943244361752704526516616394918528493321394574264607485573)$

$(-5, \quad 210855924586385522702291996875375564432834909148788263313616464995823236571191045770185801157558886696640500937)$

$(-3, \quad 22358943854775137747028532971526452088757418214703795026514539955938354527714495940754319443379591091154854811)$

$(-3, \quad-3066428918730050632325097660734163737851572230223614656315369265785635066318979992409078183621174963776878039)$

$(2, \quad 142086598987407275566528223029847683110629990793367193207487961621845231249474450762716878333452768170371307)$

$(-10,-4013833186275051054261529002448266001854462865736283000730209262386311300176819714480584897953649188768987)$

$(3, \quad 513318782779814540099933414852657264974713493469374585453379543647835185456621560862523908562876553217709)$

$(-2, \quad-61677436882563203815369113178313444194666063177138061203361907960930109397743003273918060562207208817421)$

$(4, \quad-57053438120635304110047793680658649566299800006638652578479437486915684727604688458627505624819542303243)$

$(3,-91558187817393163469667420478915357008167385723230297957370355268833319053370140794607127532476127053)$

$(21, \quad 453868533685069718784249965367088878135788567086616180536047830052899711042568402989974727201843537)$

$(-10, \quad 20964009631208954520918551253653792022251672637669648478775352234648622483831508661058580485766883)$

$(-2, \quad-2452654164796263512959165565377597147123165793392501354290866811059027722610119847271723828796771)$

$(4, \quad-147052793673461538086506530668357561094222647824631587108653738299104036416816746499164729738165)$

$(4, \quad 13963798812911120130279709463801187300299288356253613055092055278693493066888731286397000735637)$

$(-2, \quad-22430594690298603417335824980938203477805571947687436652981292220078494230642566855446565258585)$

$(3, \quad-332536984737070293356781633953414109182889100380596552532026039092003149663826893067835527979)$

$(3, \quad-538696724497089540712010373439801722161947923533835172639343996938458555469016876949250112145)$

$(2, \quad 8809243662589899724441862302427489374063110417101583652380728682887905485821254651162082773)$

$(-3, \quad 1523916850396224929683411116520822483473365693967880645688939842558533917993725763450387843)$

$(-2,-175448936439219351474943190408140984880152096488165074286001873576813341763276791632528051)$

$(4, \quad-7172119525392604758586912757900411673673727956088230007355764602327785267343320520772285)$

( $6, \quad 411983586217791325733798025629672849513780286785778486048473044819412478399711749331293)$

$(-3, \quad 399523782540463316409852453598531484804396746720468526934779408868733711190791503779555)$

$(-4, \quad 24304189642226944806712129161363218927714261648078945884775183594905868264571397226917)$

$(-4, \quad-200481638950679212651370696237549969836787003530772668084652658004782296755431254661)$

(3, -15809036670606058719969662268090458772980604046280756287155668401840558844802721257)

$(5, \quad-1523585718315717333817603063481778938222355282742962613553158680390832265726743003)$

$(2, \quad 184311493695616947777956105808291545959771200085283101706038290472860989663874931)$

$(-4, \quad 18608721351129642487382805128934749545584400507726296787447608402705855634753005)$

$(-3, \quad 1869347445173273085208023229014781878050128548111311960144791632389450110085991)$

$(-4, \quad 1783807300968884354033504909171901616894208529518268605707305999380528892537777)$

$(-3, \quad 8087485289366444463103610663066672869280303202819888635266439607722241722651)$

$(-8, \quad 330989870518604330321501498261660421116420485572033339979389384876143961861)$

$(-3, \quad-18664536615969020483601618613669413560772978432066084296155744931355960393)$

$(6, \quad-1412504875497260093376398626643530047287435788085760178403662982434702419)$

$(2, \quad 219996492734844884145785372293460505348942755871479135939553072964463795)$

$(-3, \quad 23516043605665539210119254241067982862993090461007215280854551535870327)$

$(-3, \quad-3077511624656829785194435187496372328800530833832937512976380187853603)$

$(2, \quad 297152112917428186473487402530178004938361047882953462356887529974435)$

$(-5, \quad 20430479687841138388651786490336214660319513581933064107387538439725)$ 


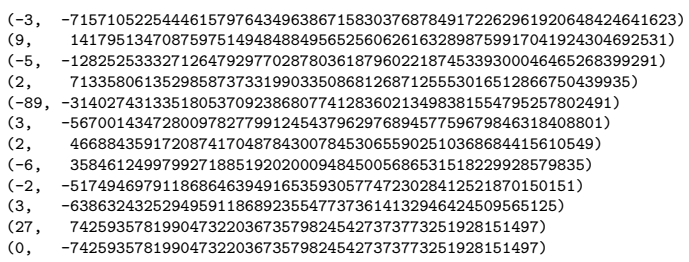

Output: the reduced ideal $(A 1, B 1)$ equivalent to $\mathfrak{A}$

$A 1=956239841432722652133553576334329186177525820778149$ $B 1=-742593578199047322036735798245427373773251928151497$ 